the university has lost what was once supposed, at least by the humanists, to give it higher meaning or identity? Readings' own solution is quixotic, given his critical stance which seems to undercut everything by ad hoc temporary norms. He proposes that the university should be a "community of dissensus", where we can talk to and accept "the Other" while exercising no hegemonic control. He recognizes that a secondary consensus would be necessary to establish such a dissensus. He does not explain, however, what the advocates of dissensus or difference are to do with "the Other" who firmly hold to an alleged norm they regard as hopelessly benighted. Readings simply tells us to accept our dependency on others, even if we cannot say why. Such a "non-finite obligation" may make some people think about religion and the mystical, he observes, "but I am not trying to sound mystical". His moral ideal, which is roughly that of the Sermon on the Mount, is admirable. How contemporary cultural criticism could persuade modern university culture to adopt it remains a mystery.

George M. Marsden is in the Department of History, University of Notre Dame, Notre Dame, Indiana 46556, USA.

\section{Two for the skies}

\author{
William H. Press
}

Blind Watchers of the Sky: The People and Ideas that Shaped Our View of the Universe. By Rocky Kolb. Addison-Wesley: 1996. Pp. 338. \$25.

Our Evolving Universe. By Malcolm S. Longair. Cambridge University Press: 1996. Pp. 185. £24.95, \$34.95.

Is basic science not getting the respect it deserves? Has 'curiosity' become a term of vilification in political quarters? While most of us in the trenches make slow progress through the pop-psych stages of denial, anger, grief and acceptance, a few of our colleagues are taking the more constructive and proactive approach of engaging the public through popular writing about basic, curiosity-driven science. Here are two books on modern cosmology, undeniably one of the purest of the basic sciences, which, in very different ways, succeed in conveying the vibrancy, creativity and sheer intellectual joy of 'this cosmology thing' and, by contagion, basic science in general.

Dr Jekyll, meet Professor Edward W. Kolb, respected author of some of the most arcane and inaccessible papers in the specialized field of particle astrophysics. $\mathrm{Mr}$ Hyde, meet Rocky Kolb, gutsy narrator of a thoroughly delightful recounting of the

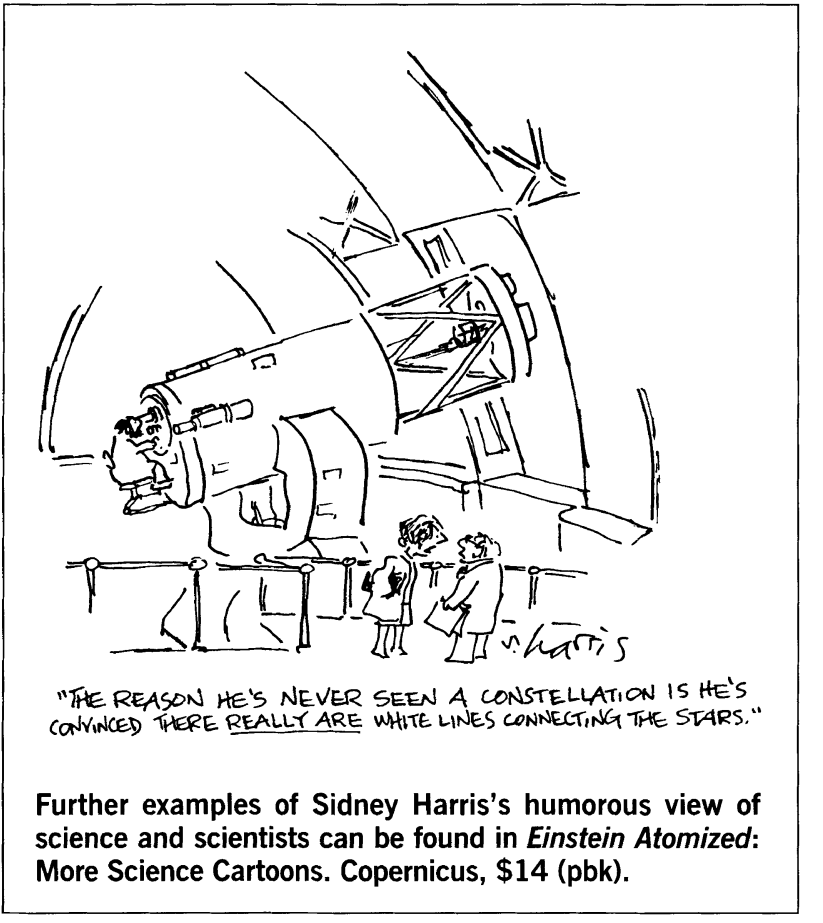

the detailed narrative focuses, as it should, on Shapley (who got it wrong), Einstein, Friedmann and Hubble (who got it right). No less interesting are such supporting players as Humason, who, without benefit of formal education beyond elementary school, rose from mule driver and construction worker to become Hubble's essential collaborator.

In the final section, Kolb introduces the cosmic microwave background and provides an account of the state of current understanding of the early moments of the hot Big Bang. Clear discussion of the science now overshadows the personalities and egos involved. One can hardly

great ideas of the past 500 years of cosmological understanding, and no less the very human personalities who have given them to us. Use of his nickname, Kolb tells us, "was to serve as a constant reminder that this book is not for the usual target audience for whom I write".

The ploy seems to have succeeded. No less successful is the daring strategy of prefacing a book on modern cosmology with 130 pages on Copernicus, Tycho Brahe, Kepler, Galileo and Newton. In the hands of 'Edward W.' this might have been dry history, but Rocky delivers (on the basis of secondary sources, but quoting liberally from the principals) a fascinating narrative, full of audacious wit and genuinely new insight, on what might have otherwise been assumed to be a thoroughly explored historical trail.

What interests Kolb, apart from tracing the history of ideas (which he does well), are questions such as "How did the personal backgrounds and eccentricities of the 'greats' affect their discoveries?" and "What parts of the modern scientific paradigm did they 'get', and which parts were they blind to?"

Kolb has a novelist's knack for projecting us backwards into epochs very different from now, and his analysis, given its generally high entertainment value, is surprisingly sophisticated. Galileo, in his view, is the only one of the bunch who could really fit right into a modern university department. (Now there's a recruitment opportunity that would make any dean drool!)

Jumping forward in time, Kolb takes up in similar vein the discovery of the Galaxy, and of the now accepted Big Bang model. A number of lesser figures have cameo roles here (Messier, the Herschels, Kirchoff, Hertzsprung and Russell) but believe that the author's gossipy interest in people (and what causes them to do good or bad science) ends with the year 1965 . Nor, I can attest, have all modern cosmologists become self-effacing specimens of the dull bourgeoisie. Can it be that Rocky is less willing to take on living targets? If so, we are deprived of some undoubtedly witty, thoughtful and provocative commentary. Perhaps another book, under a different, more clandestine, pseudonym, is in order.

Malcolm Longair's Our Evolving Universe is a more conventional, though certainly no less authoritative, introduction to modern cosmology. The author, formerly Astronomer Royal for Scotland and now a professor at the University of Cambridge, has given us a beautifully produced book, in an oversize format, with an elegant typographical design, clear diagrams and many colour illustrations. He begins by discussing some observational aspects of astronomy, then turns to the formation and evolution of stars, the origin of quasars, then galaxies and (finally) the Universe in its entirety. The text is unflinchingly direct and accurate; although there are no equations, the discussion is at a uniformly high conceptual level.

I hope that there is a market for a book like this. Its content is that of a first-rate introductory course for non-astronomy majors, while its clean format, with none of the intrusive pedagogical devices that make textbooks qua textbooks so annoying to the general reader, makes it a delight to hold.

William H. Press is at the HarvardSmithsonian Center for Astrophysics, 60 Garden Street, Cambridge, Massachusetts 02138, USA. 